\title{
La complejidad social en las estribaciones andinas orientales durante el período pre-incaico tardio
}

Catherine Lara I.

RESUMEN

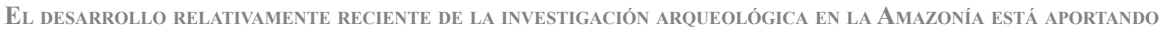
CON DATOS QUE OBLIGAN UN REPLANTEAMIENTO DE LOS MODELOS SOCIO-ECONÓMICOS TRADICIONALES ASOCIADOS AL ESCENARIO AMAZÓNICO PRECOLOMBINO EN ÉPOCAS EN QUE EL REGISTRO ARQUEOLÓGICO DE LA ZONA ERA AÚN POCO CONOCIDO. Así, EN EL CASO MÁS CONCRETO de LAS ESTRIBACIONES ANDINAS ORIENTALES, LA EVIDENCIA DESCUBIERTA SUGIERE LA PRESENCIA DE SOCIEDADES POLÍTICAMENTE COMPLEJAS, HIPÓTESIS QUE CONTRASTA CON LAS PRIMERAS PROPUESTAS REALIZADAS SOBRE LA ZONA.

Palabras claves: arqueología amazónica- estribaciones orientales, complejidad social, Verticalidad.

Abstract:

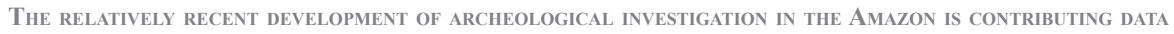

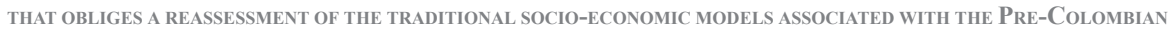
Amazon in ERAS IN WHICH THE ARCHEOLOGICAL RECORD OF THE ERA WAS STILl LiTtLe KNOWN. As SUCH, IN THE MOST CONCRETE CASE OF THE EASTERN ANDEAN FOOTHILLS, THE EVIDENCE DISCOVERED SUGGESTS THE PRESENCE OF POLITICALLY COMPLEX SOCIETIES, HYPOTHESIS THAT CONTRASTS WITH THE FIRST PROPOSALS CREATED FOR THE AREA.

KEYWORDS: AMAZONIAN ARCHAEOLOGY - EAST FOOTHILLS - SOCIAL COMPLEXITY - VERTICALITY 


\section{Introducción}

$\mathrm{L}$ os constreñimientos ecológicos e ideológicos ligados a la región amazónica la relegaron por mucho tiempo al margen de la investigación científica. En la actualidad, estos obstáculos están siendo superados; la zona, incluso, se ha convertido en un foco de estudio cada vez más decisivo en diversos ámbitos. Así, los estudios recientes relacionados a las disciplinas de la biología y la antropología han determinado que la Amazonía no constituye un espacio homogéneo.

En la arqueología andina más precisamente, la región amazónica y más particularmente la zona de estribaciones había sido tradicionalmente percibida como una periferia incorporada a núcleos políticos serranos a través de mecanismos como la verticalidad. No obstante, nuevos estudios teóricos y empíricos acerca de la formación de la complejidad social en los ecotonos están redefiniendo esta percepción. Según estos estudios, en la época precolombina, las zonas de estribación tuvieron un protagonismo que por mucho tiempo les fue denegado desde un punto de vista académico.

El siguiente trabajo se propone explorar brevemente los modelos tradicionales de verticalidad propuestos inicialmente en la explicación de los procesos culturales asociados a las áreas de estribación, antes de examinar un poco más detalladamente los primeros resultados de los nuevos avances que proponen la existencia de complejidad social en la zona, enfocándose en un caso concreto (el valle del río Cuyes, provincia de Morona Santiago). Estas consideraciones llevarán a evocar el debate sobre el origen étnico de quienes originaron esta complejidad, por lo cual se examinarán -en último término- nuevas propuestas realizadas por la etnología al respecto, con el objetivo de plantear una reflexión teórico-metodológica acerca del estudio de la problemática del origen de la complejidad social en las estribaciones orientales.

\section{La verticalidad y las estribaciones orientales andinas}

John Murra fue uno de los primeros investigadores en profundizar la noción de control de pisos ecológicos o verticalidad, en el caso de las sociedades de los Andes Centrales. Representó a la dinámica política, económica y demográfica de los núcleos poblacionales estudiados bajo la imagen de archipiélagos de islas, en los que existía un centro que se abastecía en recursos en los demás "islotes", repartidos en pisos ecológicos distintos, lo cual aseguraba una variabilidad en el aprovisionamiento de bienes (Murra, 1975: 62; Quattrin, 2001: 4).

En el actual territorio del Ecuador, Frank Salomon es quien más ha investigado acerca de la noción de verticalidad. Sus estudios en el valle de Quito y la región de Chimbo sacan a relucir que habría existido tres tipos de dinámicas económicas en las áreas investigadas: intercambio llevado a cabo por especialistas o mindalaes (en mercados posiblemente), sistema de mitmakunas (equivalente al modelo de archipiélago propuesto por Murra), e intercambio informal entre núcleos domésticos (Salomon, 1980: 181). Cabe resaltar que, en base a evidencias etnohistóricas, Salomon hace referencia al intenso nivel de contacto comercial existente entre la Sierra y la región amazónica, más particularmente entre el valle de los Quijos y el de Quito (Ibíd.: 172).

En este sentido, la modalidad "mindala" de Salomon se asemeja a la definición del puerto de comercio tal como lo concibe Hirth, como foco de contacto comercial en zonas de paso estratégicas (Hirth, 1978: 4). El poder de los caciques se deriva esencialmente aquí de su capacidad de control de las redes de intercambio, mecanismos implementados dentro del sistema vertical, y cuyas bases fueron afirmadas con la ocupación inca (Salomon, 1978). 
En este escenario, las zonas ricas en recursos no conocen un desarrollo mayor, sino que constituyen fuentes de subsistencia para los centros a los cuales se hallan subordinadas. Desde este punto de vista, Smith señala que estos puertos de comercio son la evidencia de un intercambio desigual entre lo que vendría a ser el centro y la periferia del sistema mundo tal como le define Wallerstein (Hodges, 1987: 123). Las teorías del tipo sistema mundo manejan conceptos de centros, periferias y semi-periferias, de acuerdo al grado de inclusión de las diferentes unidades dentro del sistema en cuestión. En términos generales, los centros son zonas extractoras de recursos, mientras que las periferias son las fuentes de esta extracción (Masson, 2005: 1162).

A nivel comparativo, un caso similar a los modelos anteriormente entrevistos se presenta en la región muisca (Colombia), estudiada por Langebaek (1992) desde el punto de vista de la teoría del sistema mundo, o la de Taylor (1988) y Salazar (2000) en el valle del río Cuyes (suroriente del Ecuador).

Desde esta perspectiva, Langebaek propuso que la economía muisca se organizaba en torno a un núcleo serrano del cual dependían sociedades periféricas de las tierras bajas, mediante relaciones comerciales asimétricas esenciales al mantenimiento del sistema. Así, los centros producían bienes a partir de la materia prima obtenida de estas periferias, las cuales debían estar a distancias relativamente moderadas de los núcleos políticos y económicos (Langebaek, 1992: 26).

Por su parte, Salazar y Taylor atribuyen la colonización precolombina de los valles del Cuyes y del Cuchipamba a la explotación cañari de los placeres auríferos (Salazar, 2000: 28), planteando la hipótesis de un origen cañari del registro monumental evidenciado en la zona, de acuerdo al sistema de control vertical propuesto por Taylor (Salazar, 2000: 27; Taylor, 1988: 55).

No obstante, la necesidad enunciada por el mismo Murra de afinar los parámetros de los modelos de verticalidad (Chacaltana, en prensa) y los avances en los estudios sobre los procesos de desarrollo de la complejidad social han definido una mirada distinta sobre las áreas ricas en recursos, ya no consideradas como simples "trastiendas" de los centros mayores, sino "cunas" de centros políticos autónomos.

En términos generales, los núcleos políticos surgen más particularmente en zonas claves especializadas en la producción de algún recurso y caracterizadas por la circulación de bienes a nivel local y regional, especialmente de bienes suntuosos (Hodder y Orton, 1976: 74). Son a menudo regiones que no tardan en escapar al control de centros foráneos a los que estuvieron inicialmente asociados (en este caso, serranos por ejemplo), y en convertirse ellas mismas en jurisdicciones administrativas autónomas capaces de agrupar conjuntos multiétnicos previamente dispersos (Morris, 1985: 484). Debido al papel estratégico desplegado por las zonas abundantes en recursos, Hodder plantea que éstas podrían haber favorecido el desarrollo de élites locales en base al control de los recursos en cuestión, dentro de sistemas jerárquicos de manejo de las diversas unidades territoriales (Ibíd.: 66). De esta manera, estos centros locales cobran importancia, especialmente si están en contacto con unidades políticas foráneas a través de actividades de intercambio (Ibídem: 74). Este fenómeno cobra mayor amplitud en zonas de contacto entre pisos ecológicos y etnias diversas (Ibídem: 76), las cuales favorecen las actividades de intercambio (Ibídem). De hecho, autores como Peregrine subrayan que esta forma de desarrollo de elites locales se basa no tanto en el control de redes de intercambio como en el monopolio sobre bienes de prestigio (Martin, en prensa).

De acuerdo a este escenario, las áreas más propensas a ser incorporadas como periferias en unidades políticas complejas serían luego aquellas que poseen recursos exóticos o altamente valorados, o aquellas que se encuentran en vías de acceso estratégicas (Schortman, 1987: 72). No obstante, las poblaciones locales incluidas en este tipo de sistema desarrollan a menudo jerarquías sociales que les permiten responder de forma eficiente a las exigencias del cacicazgo mayor del que dependen, y en último término, configurarse a su vez como centros de poder (Íbid: $72,75)$. 


\section{¿Complejidad social en las estribaciones orientales andinas? Un caso concreto: el valle del río Cuyes (Morona Santiago, Ecuador)}

Por su condición de frontera cultural y ecológica, las estribaciones orientales andinas constituyen un caso complejo, al ser el escenario de múltiples y numerosos procesos culturales (Cherry, 1987: 156).

Más que una zona de transición ecológica, el piedemonte andino es efectivamente una "zona intermedia entre los dos grandes universos culturales de Suramérica" (Bray, 1995: 31), lo cual explica el interés de este tipo de región, caracterizada por Bray como doble periferia: periferia del mundo andino, y periferia del mundo amazónico a la vez. Así, la autora señala la importancia de la ceja de selva, esfera de contacto imprescindible entre Sierra y Oriente (Ramírez de Jara, 1996: 68). Por mucho tiempo, la Amazonía y sus estribaciones han sido consideradas como una periferia de la civilización andina (Saulieu de, 2006: 13). Las investigaciones de Lathrap (1970), Porras (1971, 1975 a y b, 1978, 1987) y más recientemente, de Ramírez de Jara (1996) en Sibundoy (Colombia), Tamara Bray (1995) en Pimampiro, Cuellar en la región Quijos (2006), Rostain (1999), Rostoker (2005), Salazar (2000, 2004), Carrillo (2003, n/d) y Ledergerber (1995, 2006, 2007, 2008) en Morona Santiago, Saulieu (2006), Guffroy (2004) y Valdez y otros (2005) en Loja y Zamora Chinchipe, o de Ruth Shady (1999) en Perú, están contribuyendo a cuestionar esta propuesta. En este sentido, el concepto de cacicazgo, inicialmente excluido del escenario cultural precolombino amazónico, está siendo reconsiderado aquí.

El término "cacicazgo" ha sido definido de múltiples formas. Spencer y Redmond (1992), quienes trabajaron en el piedemonte de los llanos altos de Venezuela (característica que cobra luego todo su interés dentro del caso que nos ocupa aquí), lo definen en base a:

"la aparición de una jerarquía de asentamientos, la presencia de arquitectura e ingeniería monumental, un incremento considerable de la población regional, diferenciación social en las residencias y enterramientos y la presencia de relaciones sociales complejas con otras unidades políticas, incluyendo el intercambio y la guerra; estas últimas actividades fueron financiadas, parcialmente, a través de la producción de excedentes agrícolas” (Gassón, 2006: 41).

En términos generales, los cacicazgos se caracterizan por el manejo de excedentes así como tipos de especialización local en la producción de recursos diversos, utilizados también como pago a las autoridades bajo la forma de tributos eventualmente redistribuidos. Cuentan con un centro de poder, el cual incluye templos, residencias asociadas a la elite, y especialistas (Renfrew y Bahn, 1996: 168). Los templos son centros ceremoniales y rituales permanentes que representan de cierta manera el punto de referencia de toda la unidad cacical, sin llegar tampoco a configurarse como centros urbanos o administrativos. Los cacicazgos pueden ser de diversas dimensiones, pero en términos generales, se considera que su alcance demográfico abarca un rango comprendido entre los 5000 y 20000 individuos (Ibid).

Asimismo, las obras colectivas (construcción por ejemplo), son un factor que permite evaluar la capacidad de los líderes a controlar el esfuerzo de una mano de obra (Drennan, 1991: 282; Lippi, 1998: 337). Salazar (2000:72) recalca que las sociedades amazónicas contaban sin duda alguna con el grado de complejidad política suficiente para la construcción de dichas estructuras.

Con el objetivo de ilustrar de forma concreta el grado de monumentalidad -y por ende, de complejidad social- existente en las estribaciones andinas orientales, presentamos a continuación un caso concreto: los complejos arquitectónicos precolombinos del valle del río Cuyes.

Tal como lo demostraron las investigaciones pioneras de Ekstrom (1975, 1981), Taylor (1988), Salazar (2000, 2004), Ledergerber (1995, 2006, 2007, 2008) y sobre todo, Carrillo (2003, n/d) entre los años 70 y 2000 aproximadamente, el Valle del Río Cuyes (cantón Gualaquiza, Morona Santiago), se destaca por ser una zona de paso natural entre Sierra y Amazonía, que presenta una gran variedad de recursos naturales (oro por ejemplo), así como una impresionante arquitectura 
monumental, evidencia de la presencia en la zona de grupos humanos muy bien organizados, desde épocas precolombinas.

Sin embargo, fuera de los trabajos del Sr. Antonio Carrillo, quien lastimosamente no alcanzó a publicar sus datos en su integralidad, desde un punto de vista arqueológico, subsiste la duda sobre quienes construyeron las estructuras del Valle del Río Cuyes, y en qué época. En términos generales, la literatura sobre la zona menciona la presencia de Cañaris, Incas y de culturas amazónicas, pero de forma muy global y sin datos arqueológicos sistemáticos, salvo raras excepciones. A fines del 2009, auspiciado por el Instituto Nacional de Patrimonio Cultural Regional 6 y la Ilustre Municipalidad del Cantón Gualaquiza, el Proyecto Arqueológico Valle del Río Cuyes (ver Lara, 2010) se propuso comenzar a indagar sobre quiénes construyeron las estructuras del Valle del Río Cuyes, y cuándo. Este Proyecto incluyó una investigación bibliográfica detallada sobre la zona, un levantamiento topográfico de los sitios monumentales del sector, así como su excavación y el análisis de los materiales recuperados en ella (cerámica, carbón y muestras de suelo).

En total, dieciocho sitios monumentales fueron registrados en el sector, de los cuales dos desaparecieron bajo la infraestructura moderna de los pueblos de San Miguel de Cuyes y Ganazhuma (ver mapa). Los demás se podrían dividir en cuatro tipos: terrazas, pucaraes, centros ceremoniales y habitacionales.

Tres conjuntos de terrazas han podido ser registrados y mapeados aquí: 17 terrazas en Espíritu Playa (las únicas en contar con revestimiento de piedra), 35 en San Miguel de Cuyes, y 29 en Nueva-Zaruma. El análisis de muestras de suelo tomadas en dichas terrazas reveló -en dos de ellas (en San Miguel de Cuyes y Nueva Zaruma)-, huellas de plantas cultivadas, más precisamente de maíz (Zea mays), achira o platanillo (Canna sp.) y lerén o bijao (Calathea $s p$.), así como de numerosas especies leñosas y palmas, muy utilizadas en la Amazonía para la fabricación de diversos tipos de artefactos (Veintimilla, 2010).

Pasando ahora al segundo tipo de sitios aquí propuestos -los pucaráes-, cabe señalar que existen referencias etnohistóricas acerca del pasado bélico del valle del río Cuyes, siendo los protagonistas de los enfrentamientos registrados los Cañaris, los Incas (De los Ángeles, 1991; Taylor, 1988), los "Zamoranos" y sobre todo, los Jíbaros (De los Ángeles, 1991; Chacón, 1989; Carrillo, comunicación personal). En este sentido, la presencia de pucaráes en el valle del río Cuyes no extrañaría. Según los rasgos definidos por Topic (1987) en la caracterización de los pucaraes, proponemos que Trincheras y Buenos Aires corresponderían claramente a esta categoría, mientras que La Cruz, Nueva Zaruma II y Río Bravo se asociarían más bien a miradores, retomando las características propuestas por Almeida (1999: 8,9).

El sitio de Trincheras se localiza al sureste de Ganazhuma, a un kilómetro al sur del río Cuyes. Se trata de una inmensa estructura ovalada de piedra laja, de 178 metros de largo por 184 de ancho (incluyendo una profunda zanja) asentada en la loma Ganazhuma. En su extremo noreste presenta una construcción de piedra circular de 17 metros de ancho por 20 de largo que marca hoy la entrada al sitio, mientras que en su lado suroeste aparece un conjunto de muros de forma vagamente rectangular, de 26 metros de ancho por 35 de largo, con dos entradas. El sitio Buenos Aires es a su vez una estructura de tierra y piedra (basalto y cangahua), delimitada por quebradas y conformada por cuatro niveles de piedra y dos zanjas, extendiéndose el yacimiento sobre una distancia de 139 metros de largo por 69 de ancho.

Entre los miradores, La Cruz está conformado por una estructura de piedra ovalada de 18 metros de largo por 13,5 de ancho orientada en dirección noreste / suroeste, así como por tres niveles de aterrazamiento. Por su parte, Nueva Zaruma II consiste en un montículo natural de tierra bien conservado y una zanja, de 227 metros de largo por 0,95 de alto. Por último, Río Bravo forma una estructura de piedra prácticamente semicircular de 34 metros de ancho por 56,7 de largo, rodeada en su lado noreste por una zanja de 70 metros de largo, la cual se cruza a través de un "puente" de tierra. Esta zanja está separada de la estructura por una distancia de 15 metros aproximadamente. 
Cabe recordar aquí que el manejo de tipologías exige cautela, pues un solo sitio puede haber desempeñado varias funciones, ya sea desde un punto de vista sincrónico o diacrónico. En el mundo andino en general, se plantea así que los pucaraes cumplían también funciones ceremoniales (Brown Vega, en prensa), o que eran inicialmente sitios ceremoniales que fueron adaptados a usos guerreros con la llegada de los Incas, en el caso cañarí por ejemplo (Idrovo, 2004: 107). Por ende, no se descarta que Trincheras, Buenos Aires, La Cruz, Río Bravo y Nueva Zaruma II hayan estado asociados a actividades de tipo ritual pero a priori al menos, reúnen las características generales de los sitios defensivos andinos.

Existen tres sitios adicionales -La Florida, Nueva Zaruma I y Santopamba - que llaman precisamente la atención, pues reúnen parcialmente las características enunciadas por Topic aludidas más arriba, motivo por el cual se piensa que podrían corresponder también a la categoría de espacios rituales. La Florida forma efectivamente un conjunto de 109 metros de largo por 79 de ancho, con un nivel superior marcado por un recinto interno circular de piedra (canto rodado al parecer), y tres niveles más de tierra y piedra que se presentan bajo la forma de zanjas/terrazas. El sitio es de fácil acceso, por lo cual su asociación a usos defensivos es poco probable, al igual que Nueva-Zaruma I. Esta última estructura consiste en un conjunto de dos niveles de piedra y una zanja, de 57 metros de largo por 65 de ancho. Si bien Nueva-Zaruma I ofrece una vista espectacular hacia el curso inferior del río Cuyes y se asienta en una loma considerablemente empinada, contando con muros relativamente anchos, extraña que sólo tenga una zanja (que aparece entre las de menos volumen en todo el valle) y que sus dos niveles de piedra no estén separados por una trinchera sino por una plataforma de fácil acceso (entradas anchas), sin contar con la presencia de terrazas habitacionales y/o agrícolas en las cercanías. Finalmente, Santopamba es una estructura ovalada de piedra de 39 metros de largo por 17 de ancho, de dos niveles, pero sin zanja.

En la misma categoría de sitios ceremoniales -aunque no "de altura"- el sitio Playa tiene una extensión de 131 metros de largo por 88 de ancho, y se compone de cinco plataformas trapezoidales con revestimiento de piedra. Cuatro de estas estructuras cuentan con muros de piedra que salen de su lado oeste ( 8 muros en total) y se dirigen hacia el noroeste del yacimiento.

Finalmente, los sitios Espíritu Playa, El Cadi, y quizá Santa Rosa se inscribirían en la categoría de sitios habitacionales.

El conjunto de Espíritu Playa - de dimensiones reducidas- cuenta con un recinto formado por tres muros contiguos a una estructura más pequeña dividida en dos cuartos, así como un empedrado. El material de construcción es el canto rodado. Por otra parte, Santa-Rosa -sector bajo de las terrazas de San Miguel de Cuyes- es un recinto de piedra laja de 26 metros de largo por 20 de ancho, dividido en dos cuartos y que presenta una zanja en su extremo noroeste. En fin, con sus 108.402 metros cuadrados de superficie, El Cadi es sin duda alguna el sitio más impresionante y complejo del valle, al presentar una estructura redonda, un recinto ovalado, una plataforma, 16 cuartos rectangulares y 24 muros de vastas dimensiones.

Un sitio que escapa a esta propuesta tipológica es el de San Juan de Kayamás, ubicado en la margen derecha del río Cuyes (en su parte baja), a pocos metros de la orilla del mismo, en un espacio relativamente plano aunque caracterizado por la presencia de una leve pendiente al sur del complejo. Este yacimiento presenta un área aproximada de 55.038 metros cuadrados, cubierta de montículos de tierra forrados de piedra de río repartidos de manera irregular. Debido a la extensión de este conjunto y al tiempo limitado del que se disponía en el marco del proyecto, no se alcanzó sino a levantar el plano topográfico de 87 de estas formaciones, de las cuales 6 se distinguen por presentar una forma alargada que recuerda la de algún muro. Los montículos se caracterizan por un largo de 2.2 metros, un ancho de 2.4 metros y un alto de 40 $\mathrm{cm}$ aproximadamente. Existen diversas hipótesis acerca de la naturaleza de estas formaciones: material proveniente de actividades de explotación aurífera (Temme, comunicación personal) 
o sencillamente construcciones (Sarmiento, comunicación personal). El material hallado en la excavación de este yacimiento abogaría más bien por su asociación a la explotación de placeres auríferos.

Desde un punto de vista cronológico, dos épocas de ocupación de las estructuras se pueden definir claramente aquí en base a las fechas radiocarbónicas obtenidas en el proyecto: la primera, muy temprana (periodo Formativo), se ubica en la parte alta del valle, mientras que la segunda, tardía (quizá desde el siglo XIV de nuestra era), la encontramos principalmente en la parte baja.

El sector asociado a las dataciones antiguas obtenidas corresponde a las inmediaciones de San Miguel de Cuyes (sitios Santa Rosa y Playa), las cuales eran aparentemente habitadas ya en el primer milenio antes de Cristo. La cerámica encontrada aquí correspondería al estilo Tacalshapa III, de acuerdo a la caracterización propuesta por Idrovo (2000: 57).

El segundo momento cronológico resaltado por las fechas radiocarbónicas aparece en la parte baja del valle, más concretamente en el sitio de Nueva Zaruma I (sector de Nueva Tarqui), hacia el siglo XIV de nuestra era aproximadamente. Al parecer, esta época coincide con el complejo de terrazas identificado en la zona, y más particularmente con prácticas de cultivo evidenciadas allí. Nueva Zaruma I es paulatinamente abandonada, mientras que Nueva Zaruma II parece revelar una ocupación humana más importante en épocas tardías, asociada quizá a la de San Juan. En el siglo XV de nuestra era en cambio, se produce una "ola" de monumentalidad, asociada a la construcción de La Florida, probablemente relacionada a la de El Cadi y Río Bravo, tal como lo indican las similitudes entre la cerámica y la arquitectura de estos tres complejos. Las fechas no son tan precisas, pero el surgimiento de Buenos Aires no parece estar alejado de este despegue del sector El Cadi. Por otro lado, en la parte alta, aparecen las estructuras de Espíritu Playa y La Cruz, cuya cerámica difiere notoriamente de la de El Cadi y Buenos Aires, indicando probablemente un origen poblacional distinto. Un porcentaje considerable del material cerámico recuperado en la parte baja del valle correspondería a la tradición corrugada (según la caracterización propuesta por Valdez, 2009: 52); se identificaron también fragmentos posiblemente asociados a algún tipo de cerámica local.

Es inevitable resaltar que el despliegue de monumentalidad observado en el lapso de 1410-1630 evidenciado por las fechas radiocarbónicas, se da precisamente en un contexto particularmente agitado en el Austro del actual territorio del Ecuador, tal como lo revelan las fuentes etnohistóricas: guerras incaicas, conquista española y subsiguientes movimientos migratorios. Si bien la correspondencia de fechas va en el sentido de una asociación entre el valle del río Cuyes y este contexto, los datos disponibles por el momento no permiten definir precisamente a cual o cuales de estos eventos está vinculada el área en cuestión.

\section{Aportes de la etnología}

En términos generales -y volviendo a un enfoque regional- se presenta luego un escenario en que aparece una presencia obvia de monumentalidad en las estribaciones orientales andinas, lo cual descarta de entrada las hipótesis deterministas que atribuyen la ausencia de grupos políticos complejos en la Amazonía a la hostilidad del entorno natural. No obstante, subsisten cuestionamientos acerca del origen étnico de quienes originaron esta monumentalidad y por ende, que protagonizaron esta manifestación de complejidad social.

¿Se trata acaso de grupos amazónicos? ¿Serranos? ¿De etnias asociadas a mestizajes entre grupos serranos y amazónicos? O por último, ¿se dieron estos tres escenarios en episodios distintos? En el valle del río Cuyes por ejemplo, se ha propuesto un posible origen inca de las estructuras (en Taylor, 1988: 38), lo cual evoca el debate acerca de la presencia inca en la Amazonía en general. Las pocas investigaciones llevadas a cabo en ese sentido dejan a entender que los Incas estuvieron poco presentes en la zona, especialmente debido a su difícil acceso y la hostilidad de las poblaciones locales (Hirschkind, 1995: 23), y que privilegiaron más bien 
estrategias de alianzas (Berthelot, 1986: 69,71; Pärssinen y Siiriäinen, 2003: 83, 103) y/o de intercambio (Oberem, n/d: 202, 206) con las poblaciones locales.

Los hallazgos de la arqueología amazónica y la perspectiva subsiguiente de la existencia de formas de complejidad social en el Oriente precolombino se compaginan con aportes recientes realizados por la etnología y más concretamente, los estudios de Phillipe Descola (2005), retomados por Saulieu (2006) desde un punto de vista arqueológico.

Así, Descola propone que existen cuatro formas generales de percibir el cosmos: el animismo, el totemismo, el naturalismo y el analogismo. El primero establece un parecido de interioridades entre los seres, pero una diferencia en los físicos, en oposición al naturalismo. Por otro lado, el totemismo plantea un parecido de interioridades y físicos, contrariamente al analogismo, que razona en función a una diferencia entre estos dos últimos elementos (Descola, 2005: 176). El animismo así definido sería recurrente en las culturas aborígenes de América del Norte y en las de foresta tropical, mientras que el analogismo se encontraría en el mundo andino (Ibíd.: 289). Según esta propuesta, las estribaciones orientales andinas se ubicarían luego en una frontera entre el pensamiento analógico y animista. El pensamiento analógico se caracteriza precisamente por la representación de un "afuera" compuesto por "bárbaros" eventualmente susceptibles de ser incorporados al orden analógico. Aquí, Descola da el caso del la Amazonía, en donde esta relación entre animismo y analogismo parece darse precisamente según dinámicas distintas dependiendo de los grupos culturales en contacto y de la naturaleza de su coexistencia (Íbid).

Explorando las implicaciones del modelo de Descola a nivel del registro arqueológico, de Saulieu (2006) propone justamente un escenario cultural hipotético para la época precolombina en la Amazonía, el cual divide en dos etapas.

En la primera, que abarca los periodos Formativo y de Desarrollo Regional (3500 - 300 a.C.), la Alta Amazonía se habría desarrollado a la par de sus vecinos de los Andes Centrales y Septentrionales, en estrecha relación con ellos. Es así como en la segunda fase de este proceso, las culturas de la alta Amazonía se habrían caracterizado por la producción de arquitectura monumental.

En una segunda etapa, la expresión de influencias locales y de grupos culturales nuevos emparentados a las sociedades amazónicas rastreadas gracias a la evidencia etnohistórica, da un curso distinto a las dinámicas culturales del sector. Según de Saulieu, el pensamiento animista tal como lo describe Descola llega poco a poco a dominar en la zona, acarreando una serie de consecuencias en toda la escala de las manifestaciones culturales y desde luego, en el registro arqueológico de la alta Amazonía. Entre estas consecuencias, desaparece la monumentalidad y se descentraliza el patrón de asentamiento. No obstante, hubo al parecer excepciones, que son de particular interés para nuestro caso de estudio:

"El otro escenario que se da, muestra a lo largo de ciertos ríos navegables y en ciertos sectores de las vertientes andinas (valle de los Quijos), la disposición de sistemas sociales complejos, fundamentados en el intercambio comercial. El Horizonte Polícromo procedente de las llanuras aluviales de la Baja Amazonía coloniza, hacia los siglos X y XI, el Napo y una parte del Marañón, hasta el Putumayo, y se articula con el Panzaleo-Cosanga-Píllaro sobre la vertiente andina. Los datos etnohistóricos nos permiten reconocer los diversos grupos étnicos y muchas veces de origen lejano, los cuales tienen funcionamientos sociales complejos y jerarquizados, con sistemas de producción especializada (particularmente el oro, la cerámica y los tejidos de algodón). Los intercambios a través de grandes distancias, se concentran en algunos grandes ejes formados por el Napo, el Marañón, el Ucayali y el Huallaga (sal, curare, oro, algodón, aceite de tortuga, etc.) y parecen controlados por estas poblaciones que tienen una inclinación fuerte para la navegación y el intercambio fluvial" (Saulieu de, 2006: 20). 
En ese sentido, la ceja de montaña y su riqueza natural y cultural serían algo así como un punto de encuentro entre mundo andino y cosmovisión amazónica, que definió los procesos posteriores acaecidos en ambos espacios (Valdez, comunicación personal). El sitio Santa Ana La Florida, en el cantón Palanda (Zamora-Chinchipe), es asimismo contemporáneo de Valdivia (Valdez et al., 2005: 374), y presenta huellas de monumentalidad inclusive más desarrolladas que en Valdivia.

\section{Observaciones finales: hacia una propuesta teórico-metodológica}

Es ya un hecho: el medio de estribación no es un obstáculo al desarrollo de grupos políticamente complejos; los hallazgos cada vez más numerosos de conjuntos arquitectónicos monumentales precolombinos en la zona dan cuenta de ello. No obstante, como se vio, el tema de la complejidad va de la mano con la problemática del origen étnico de quienes levantaron estas estructuras.

Desde este punto de vista, la diversidad cultural de la Amazonía, incluyendo sus partes altas, no va en el sentido de la propuesta de un solo escenario cronológico y cultural de poblamiento, ni por ende, de un solo modelo político aplicable a cada uno de los conglomerados sociales que habitaron las estribaciones orientales andinas. A lo sumo, se puede llegar a un panorama general en que cada uno de estos conglomerados sociales presenta un caso particular (lo cual no excluye similitudes o vínculos entre ellos), a la manera de un mosaico cronológico y geográfico que le corresponde a la investigación arqueológica reconstruir desde una perspectiva inductiva deductiva. Deductiva, pues cualquier estudio que se realice sobre el tema en una zona precisa no debe perder de vista el enfoque regional, y deductiva, pues los aportes realizados en la zona en cuestión contribuirán a su vez al conocimiento de este "mosaico".

Así, cualquier investigación específica acerca de alguna de las agrupaciones políticas que poblaron las estribaciones orientales exige antes que nada el establecimiento de la identificación étnica y cronológica de los actores en juego. ¿Correspondieron estos a poblaciones serranas del Formativo (como fue posiblemente el caso en San Miguel de Cuyes)? ¿Amazónicas (como en el caso de Santa Ana - La Florida -formativo amazónico)? ¿A grupos mestizados "serranoamazónicos" (como en las sociedades precolombinas de estribación investigadas en Perú por Pärssinen y Siiriäinen (2003: 104, 106), o eventualmente la parte baja del valle del río Cuyes)? ¿Se dieron acaso estas tres posibilidades en épocas distintas?

De acuerdo a Descola, el pensamiento analógico (generalmente asociado a lo andino) se caracteriza precisamente por la búsqueda de orden (2005: 547), lo cual otorga a su vez un papel clave a las estructuras de poder y a sus diversas manifestaciones (tales como la monumentalidad). Si bien el pensamiento animista no percibe el poder de la misma forma, Descola no descarta que la diversidad de las sociedades amazónicas y los numerosos casos de mestizaje haya originado excepciones en este sentido (2005: 289). La monumentalidad evidenciada en la Amazonía permite de hecho hablar de una "complejidad amazónica". La propuesta de Saulieu cobra todo su sentido aquí, al proponer precisamente la existencia de una monumentalidad amazónica formativa y luego, en las zonas de estribación, aunque se trata de un escenario que amerita aún ser más ampliamente comprobado en el registro arqueológico.

Sólo con el conocimiento preciso del origen étnico de los actores en juego y de su contexto cronológico es posible plantearse problemáticas relacionadas a la naturaleza de los procesos políticos propios a la complejidad reflejada por el registro. Aquí también se perfilan "posibilidades": verticalidad, autonomía política, procesos "mixtos" (verticales y autónomos) desde un punto de vista diacrónico... 
En el caso del Cuyes, la evidencia recopilada de momento iría más bien en el sentido de una ocupación de tipo vertical en épocas muy tempranas (formativo), y luego, de una autonomía política protagonizada por un grupo étnico "mestizo" amazónico y probablemente serrano. Este escenario no es más que una suposición, pues falta todavía investigación en la zona para esclarecer totalmente el escenario étnico y cronológico del valle.

En resumidas cuentas, queda claro que en términos generales, las estribaciones orientales andinas no fueron una simple periferia del mundo andino, sino un espacio que jugó un papel clave en las distintas etapas de su formación, no solamente debido a su riqueza en materias primas, sino también como región fronteriza entre Sierra y Amazonía. El conocimiento del pasado precolombino de la zona aportará sin duda con elementos claves para el entendimiento de la formación del mundo andino y la evolución de la complejidad social en general, por lo cual el desarrollo de investigaciones más profundizadas en el área es un fundamental tanto desde un punto de vista científico como patrimonial.

\section{Bibliografía}

Almeida Reyes, Eduardo. 1999, Estudios arqueológicos en el pucará de Rumicucho. II Etapa, Museo del Banco Central del Ecuador, Quito.

Berthelot, Jean. 1986, "The extraction of precious metals at the times of the Inka", in Anthropological history of Andean polities, John V. Murra; Nathan Wachtel, Jacques Revel eds., Cambridge University Press \& Éditions de la Maison des Sciences de l'Homme, United-States, pp. 69-88.

Bray, Tamara. 1995, "Pimampiro y puertos de comercio: investigaciones arqueológicas recientes en la Sierra Norte del Ecuador", in Perspectivas regionales en la arqueología del Suroccidente de Colombia y Norte del Ecuador, Cristóbal Gnecco, ed., Editorial Universidad del Cauca, Popayán, pp. 30-48.

Cárdenas, Bolívar. 2004, Caciques cañaris. CCE Benjamín Carrión, Núcleo del Cañar. Azogues.

Carrillo, Antonio. 2003, "El Señorío de los Cuyes", in Cuenca ilustre. N/d.

Chacaltana Cortez, Sofia; Christopher Dayton; Mónica Barrionuevo. "Sistemas de almacenamiento en la costa y la sierra de Colesuyo, Andes Centrales" in Comparative Perspectives about the Archaeology of Coastal South America, Alexander Martín; Enrique López-Hurtado; Robyn E. Cutright eds., University of Pittsburgh Latin American Archaeology Publications, (en prensa).

Chacón, Juan. 1989, "Historia de las minas de oro y plata, de la antigua provincia de Cuenca", in La sociedad azuayo-cañari: pasado y presente, Leonardo Espinosa editor, Editorial El Conejo. Quito.

Cherry, J.F. 1987, Power in space: archaeological and geographical studies of the State", in Landscape and culture: geographical and archaeological perspectives, J.M. Wagstaff ed., Basil Blackwell, Londres, pp. 146-172.

Cuellar, Andrea. 2006, The organization of agricultural production in the emergence of chiefdoms in the Quijos region, Eastern Andes of Ecuador. Disertación de PhD, Universidad de Pittsburgh. Pittsburgh.

De los Ángeles, Domingo. 1991, "Relación que envió a mandar su majestad se hiciese de esta ciudad de Cuenca y de toda su provincia”, en: Ponce Leiva, Pilar (ed.) Relaciones histórico-geográficas de la Audiencia de Quito (siglos XVI-XIX), tomo I, Consejo Superior de Investigaciones Científicas, Centro de Estudios Históricos, Departamento de Historia de América, Madrid, pp. 372-406.

Descola, Philippe. 2005, Par-delà nature et culture, Ediciones Gallimard, París.

Drennan, Robert. 1991, «Pre-hispanic chiefdom trajectories in Meso-America, Central America and northern South-America», en: Timothy Earle (ed.) Chiefdoms: power, economy and ideology, School of AMerican Research / Advanced Seminar Series, Cambridge, pp.263-287 .

Ekstrom, Peter J. 1975, "Responding to a new ecology: adaptations of colonists in eastern Ecuador", en: Papers in Anthropology, vol. 16, $\mathrm{n}^{\circ}$. Department of Anthropology.

1981, "Colonist Strategies of verticality in an eastern valley", en: Cultural Transformations and ethnicity in modern Ecuador, Norman Whitten ed., University of Illinois Press, Estados-Unidos, pp. 327-355. 
Gassón, Rafael A. 2006, "Los sabios ciegos y el elefante: sistemas de intercambio y organizaciones sociopolíticas en el Orinoco y áreas vecinas en la época prehispánica", in Contra la tiranía tipológica en arqueología, Cristóbal Gnecco; Langebaek Karl eds., Uniandes-CESA, Bogotá, pp. 31-53.

Guffroy, Jean. 2004, Catamayo precolombino, UTPL, IFEA-IRD Éditions. París.

Hirshkind, Lyn. 1985, "Cañar incásico", in Universidad Verdad, Universidad del Azuay, Cuenca, pp. 15-54.

Hirth, Kenneth. G. 1978, "Interregional trade and the formation of prehistoric gateway communities", in American Antiquity, vol. 43, No. 1, January, pp. 35-45.

Hodder, Ian; Clive Orton. 1976, Spatial analysis in archaeology, Cambridge University Press. Cambridge.

Hodges, R. 1987, "Spatial models, anthropology and archaeology", in Landscape and culture: geographical and archaeological perspectives, J.M Wagstaff ed., Basil Blackwell, Londres, pp. 118-133.

Idrovo, Jaime. 2000, Tomebamba: arqueología e historia de una ciudad imperial, BCE, Dirección Cultural Regional Cuenca. Cuenca.

Aproximaciones a la historia antigua de la bio-región del Chanchán, Municipalidad de San Pedro de Alausí. Quito, noviembre del 2004.

Langebaek, Carl. 1992, Noticias de caciques muy mayores. Origen y desarrollo de las sociedades complejas en el nororiente de Colombia y norte de Venezuela, Universidad de los Andes, Bogotá.

Lara, Catherine. 2010, "Proyecto Arqueológico Valle del Río Cuyes: informe final", entregado al INPC Regional 6. Cuenca, febrero del 2010.

Lathrap, Donald W. 1970, The Upper Amazon, Thames \& Hudson. Londres.

Ledergerber-Crespo, P. 1995, "Factores geográficos en la localización de sitios arqueológicos", en: Guinea Mercedes y Jean-François Bouchard (eds.) Cultura y medio-ambiente en el área septentrional andina, AbyaYala, Cayambe, pp. 343-375.

2006 ,"Ecuador Amazónico-Andino: Apropiación de Paisajes y Relaciones Culturales”, en Pueblos y Paisajes Antiguos de la Selva Amazónica, G. Morcoter Ríos, S. Mora Camacho y C. F. Calvo (Ed.). Pp.131-155. Bogotá: Univ. Nacional,

2007, "Investigaciones arqueológicas en los valles del Cantón Gualaquiza (Provincia de Morona-Santiago)", in www.arqueo-ecuatoriana.ec

"Sur Oriente Ecuador: apropiación de paisajes a partir del Periodo Formativo Temprano", in Miscelánea Antropológica Ecuatoriana, Segunda Época, Año $1-\mathrm{N}^{\circ} 1$, Boletín de los Museos del Banco Central del Ecuador. Guayaquil, 2008.

Lippi, Ronald. 1998, Una exploración Arqueológica del Pichincha Occidental, Ecuador, Museo Jacinto Jijón y Caamaño. Quito.

Malengreau, Jacques. 1995, Sociétés des Andes: des empires aux voisinages, ediciones Karthala. Paris.

Martín, Alexander; Enrique López-Hurtado; Robyn E. Cutright. "Comparative Perspectives: an introduction", in Comparative Perspectives about the Archaeology of Coastal South America, Alexander Martín; Enrique López-Hurtado; Robyn E. Cutright eds., University of Pittsburgh Latin American Archaeology Publications, (en prensa).

Masson, Marylin A. 2005, «Trade and exchange », in Archaeological methods vol.2, ed. Herbert D.G. Maschner y Christopher Chippirdale eds., Altamira Press, pp. 1138-1178. Estados-Unidos.

Morris, Craig. 1985, "From principles of ecological complementarity to the organization and administration of Tawantinsuyu", in Andean ecology and civilisation: an interdisciplinary perspective on andean ecological complementarity, University of Tokyo Press, pp. 477-490. Tokyo.

Murra, John V. 1975, Formaciones económicas y políticas del mundo andino, Institutos de Estudios Peruanos. Lima.

"The expansion of the Inca state: armies, war and rebellions", in Anthropological history of Andean polities, John V. Murra; Nathan Wachtel, Jacques Revel eds., Cambridge University Press \& Éditions de la Maison des Sciences de l'Homme, pp. 49-58. Cambridge, 1986.

Oberem, Udo. Los Quijos: historia de la transculturación de un grupo indígena en el Oriente ecuatoriano, Instituto Otavaleño de Antropología. Otavalo, n/d.

Pärssinen Martti; Ari Siiriäinen. 2003, Andes Orientales y Amazonía Occidental, ensayos entre la historia y la arqueología de Bolivia, Brasil y Perú, Producciones CIMA. La Paz.

Ponce Leiva, Carmen Rosa. 1975, "Estudio sobre los Cañaris", documento de la Biblioteca del Banco Central del Ecuador (código: CU010040). Quito. 
Porras, Pedro. 1971, Reseña histórica de las investigaciones arqueológicas en el Oriente ecuatoriano. Ecuatoriana, Quito.

1987. Investigación arqueológica en las faldas del Sangay, Artes Gráficas Señal, Quito.

1978. Arqueología de la Cueva de los Tayos PUCE. Quito

1975. Fase Cosanga, PUCE. Quito.

1975b. Fase Pastaza, PUCE. Quito.

Quattrin, Dale; W. Uribe. 2001, Prehispanic chiefdoms in the valle de la Plata, vol 4, Vertical economy, interchange and social change during the Formative Period, University of Pittsburgh/Universidad de los Andes. Pittsburgh/Bogotá.

Ramírez de Jara, María-Clemencia. 1996, Frontera fluida entre Andes, Piedemonte y Selva: el caso del valle de Sibundoy, siglos XVI-XVIII, Instituto Colombiano de Cultura Hispánica. Bogotá.

Renfrew, Colin; Paul Bahn. 1996, Archaeology: theories, methods and practices, Thames \& Hudson. EstadosUnidos.

Rostain, Stéphen. 1999, "Excavación en área en un montículo de Huapula (proyecto Sangay Upano)”, en: Memorias del Primer Congreso ecuatoriano de Antropología vol. VIII, Simposio de Arqueología, Ernesto Salazar ed., Museo Jacinto Jijón y Caamaño, pp. 227-256. Quito.

Rostoker, A. 2005, Dimensions of prehistoric human ocupation in the southern Ecuadorian Oriente, vol. 1. Disertación de PhD, Universidad de Nueva-York.

Salazar, Ernesto. 2000, Pasado precolombino de Morona-Santiago, Casa de la Cultura Ecuatoriana Núcleo Morona-Santiago. Macas.

"Cuenca y su región: en busca del tiempo perdido", in Cuenca, Santa-Ana de las Aguas, Ed. Libri-Mundi pp. 19-85. Quito, 2004.

Salomon, Frank. 1978, Ethnic Lords of Quito in the age of the Incas: the political economy of North-Andean chiefdoms, a thesis presented to the faculty of the graduate school of Cornell University in Partial Fulfillment for the Degree of Doctor of Philosophy, 1980, Los señorios étnicos de Quito en la época de los Incas, IOA. Otavalo.

Saulieu de, Geoffroy; Lino Rampón Zardo. 2006, Colección arqueológica de Morona-Santiago del Museo Amazónico de la UPS. Una introducción a la Amazonía ecuatoriana prehispánica, Abya-Yala. Quito.

Schortman, Edward M.; Patricia A. Urban. 1978, "Modeling interregional interaction in prehistory", en: Advances in archaeological method and theory, vol.1, Michael B. Schiffer ed., Academic Press, pp. 37-95. EstadosUnidos.

Shady, Ruth. 2000, "Sociedades formativas de Bagua-Jaén y sus relaciones andinas y amazónicas", in Formativo Sudamericano, Ledergerber-Crespo Paulina ed., Abya-Yala, pp. 201-211. Quito.

Spencer, Charles; Elsa Redmond. 1992, "Prehispanic chiefdoms of the western Venezuelan Llanos", in World Archaeology 24: 134-157.

Taylor, Anne-Christine. 1988, Al este de los Andes, t.II, Abya-Yala. Quito.

Topic, John; Theresa Lange Topic. 1987, "The Archaeological Investigation of Andean Militarism: Some Cautionary Observations", in The Origins and Development of the Andean State, editado por J. Haas, T. Pozorski and S. Pozorski, pp. 47-55. Cambridge University Press, Cambridge.

Valdez, Francisco. 2009, "Informe Final de los trabajos arqueológicos realizados en el marco del proyecto UTPL-IRD / Ministerio de la Cultura", En Informe Final del Proyecto Investigación y puesta en valor de los recursos patrimoniales en la Frontera Sur, Palanda, Provincia de Zamora Chinchipe", Presentado por la UTPL al Ministerio de Cultura.

Valdez, Francisco; Jean Gufroy; Geoffroy de Saulieu; Julio Hurtado; Alexandra Yépez. 2005, Découverte d'un site cérémoniel formatif sur le versant oriental des Andes. En www.ird.fr

Veintimilla, César. 2010, Informe "Análisis de fitolitos en terrazas agrícolas del valle del río Cuyes". Guayaquil. 
LA COMPLEJIDAD SOCIAL EN LAS ESTRIBACIONES ANDINAS ORIENTALES | 89

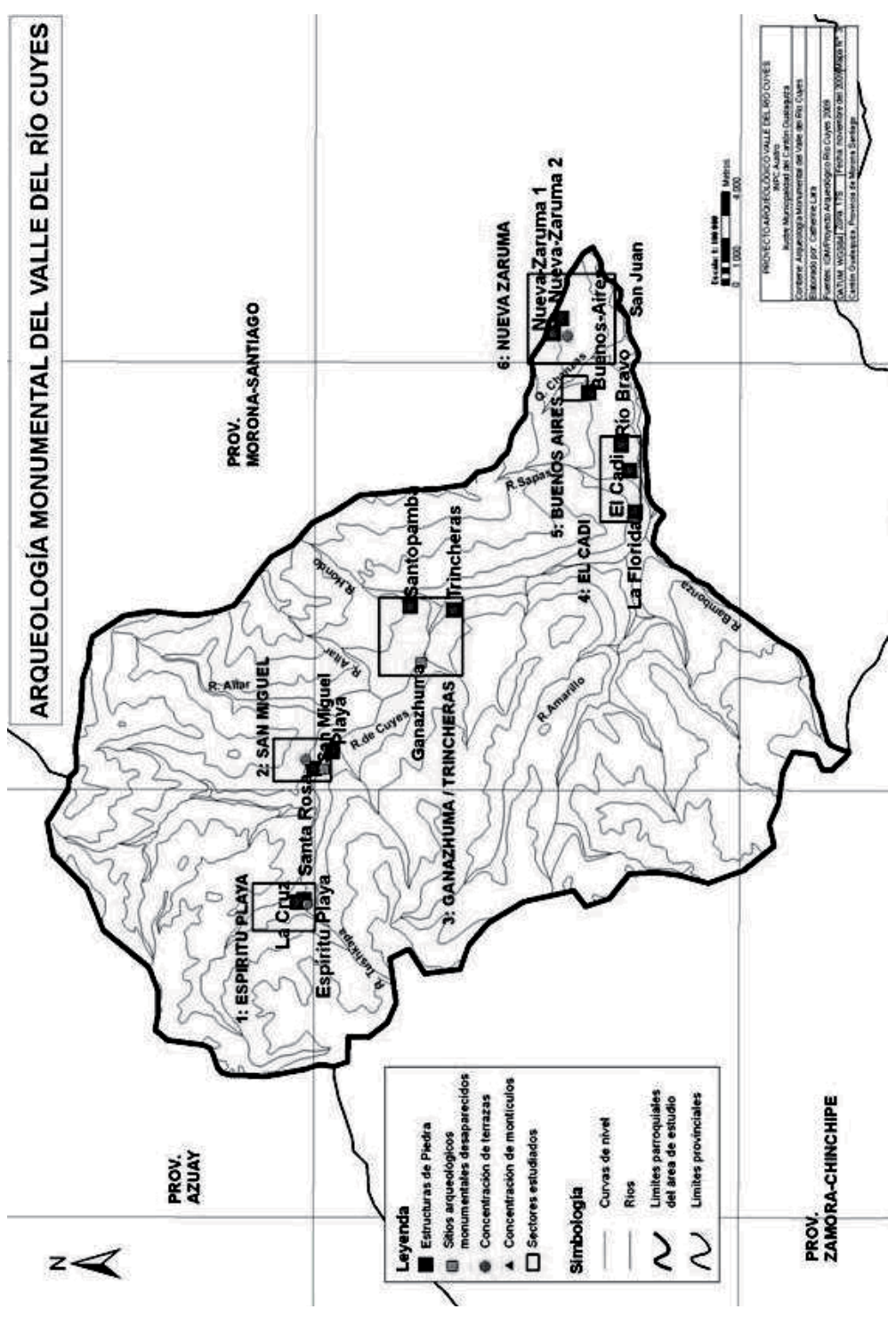




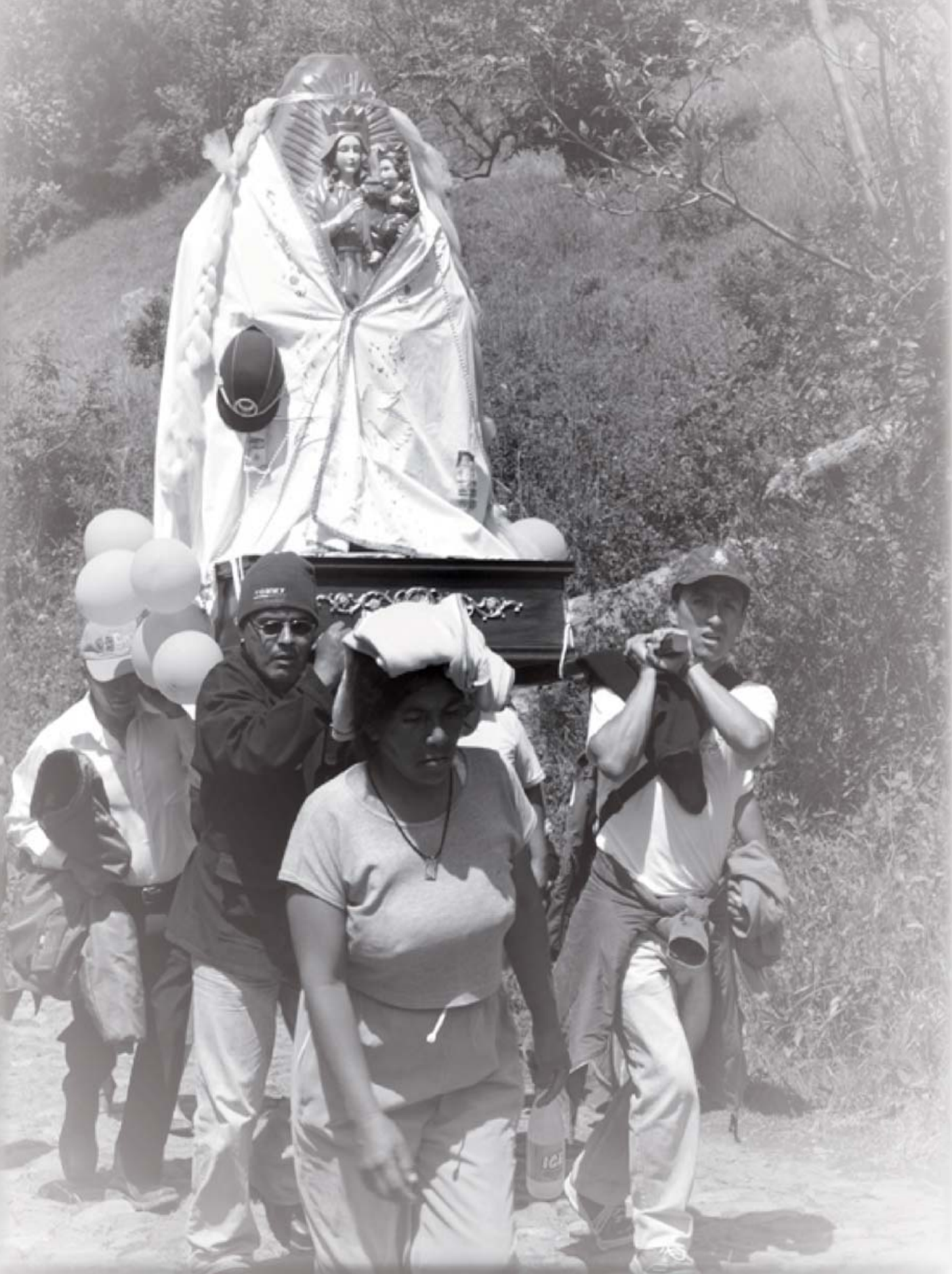

